\title{
Use of Relaxation Techniques and Complementary and Alternative Medicine by American Adults with Insomnia Symptoms: Results from a National Survey
}

\author{
Suzanne M. Bertisch, M.D., M.P.H. ${ }^{1}$; Rebecca Erwin Wells, M.D., M.P.H. ${ }^{2 ;}$ Michael T. Smith, Ph.D.; Ellen P. McCarthy, Ph.D., M.P.H. ${ }^{4}$ \\ ${ }^{\prime}$ Divisions of General Medicine and Primary Care, and Pulmonary, Critical Care, and Sleep Medicine, Department of Medicine, Beth \\ Israel Deaconess Medical Center, Harvard Medical School, Brookline, MA: ${ }^{2}$ Department of Neurology, Beth Israel Deaconess Medical \\ Center, Harvard Medical School, Boston, MA; ${ }^{3}$ Department of Psychiatry and Behavioral Sciences, Johns Hopkins University School of \\ Medicine, Behavioral Sleep Medicine Program, Johns Hopkins Bayview Medical Center, Baltimore, MD; ${ }^{4}$ Division of General Medicine \\ and Primary Care, Department of Medicine, Beth Israel Deaconess Medical Center, Harvard Medical School, Brookline, MA
}

Introduction: Though relaxation training is recommended for insomnia, national patterns of use remain unknown. Similarly, rates of complementary and alternative medicine (CAM) use by adults with insomnia are not well established. We sought to elucidate the patterns and reasons for use of relaxation techniques and CAM use by adults with insomnia symptoms.

Methods: We used the 2007 National Health Interview Survey ( $n=23,358$ ) to estimate prevalence of use among adults by self-reported insomnia symptom status. Among adults reporting insomnia symptoms $(n=4,415)$, we examined reasons for use and disclosure to medical professionals. We employed logistic regression to determine the adjusted associations between relaxation techniques use, CAM use, and insomnia symptoms.

Results: Among adults with insomnia symptoms, 23\% used relaxation techniques and $45 \%$ used CAM annually. After adjustment, adults with insomnia symptoms had higher likelihood of using relaxation techniques (aOR 1.48, 95\% Cl 1.32, 1.66) and CAM (aOR 1.29, 95\% $\mathrm{Cl} 1.15,1.44)$ compared with adults without insomnia. Deep breathing exercise was the most com- monly used relaxation technique. Fewer than $2 \%$ of adults with insomnia used CAM specifically for insomnia. Only $26 \%$ of adults with insomnia symptoms disclosed their relaxation techniques use to medical professionals. Being male, lower educational and physical activity levels, income $<\$ 20,000$, living in South, and hypertension were associated with lower likelihood of relaxation techniques use among adults with insomnia symptoms.

Conclusion: While adults with insomnia symptoms commonly use relaxation techniques and CAM, few are using for their insomnia. Facilitating discussions about relaxation techniques may foster targeted use for insomnia.

Keywords: Insomnia, relaxation techniques, complementary therapies, behavioral therapies, United States, epidemiology, NHIS

Citation: Bertisch SM; Wells RE; Smith MT; McCarthy EP. Use of relaxation techniques and complementary and alternative medicine by American adults with insomnia symptoms: results from a national survey. J Clin Sleep Med 2012;8(6):681-691.
A

pproximately $10 \%$ to $15 \%$ of adults suffer from chronic insomnia. ${ }^{1}$ Both central and autonomic nervous system hyperarousal have been strongly implicated in the pathophysiology of insomnia, ${ }^{2,3}$ and, as such, relaxation techniques have been explored as treatment for insomnia for several decades. ${ }^{4-6}$ Relaxation training comprises a diverse group of practices (e.g., progressive muscle relaxation, diaphragmatic breathing) to reduce "somatic tension" or "intrusive thoughts." When employed during pre-sleep periods, these therapies are thought to counteract the cognitive and physiologic mechanisms that initiate and perpetuate insomnia, and are recommended as "standard" treatment for insomnia by the American Academy of Sleep Medicine (AASM). ${ }^{7}$ However, little is known about the prevalence, patterns, and reasons for use of relaxation techniques by adults with insomnia.

While conventional pharmacologic and behavioral treatments can be effective for insomnia, many patients with insomnia report seeking alternative therapies for sleep complaints. ${ }^{8}$ The National Institutes of Health defines complementary and

\section{BRIEF SUMMARY}

Current Knowledge/Study Rationale: Although relaxation techniques (RT) and complementary and alternative medicine (CAM) approaches are commonly used in the United States, and practice guidelines recommend RT as a "standard treatment" for insomnia, neither RT nor CAM usage among adults with insomnia is well understood. We therefore investigated national usage patterns of both RT and CAM among adults with insomnia symptoms.

Study Impact: We found that adults with insomnia symptoms have high prevalence of use of RT and CAM, though specific use for insomnia was uncommon. Further investigations to determine barriers to use of relaxation techniques for insomnia are warranted.

alternative medicine (CAM) as a group of diverse medical and health care systems, practices, and products that are not generally practiced by allopathic physicians and allied health professionals. ${ }^{9}$ One study estimated 1.6 million Americans used CAM to treat insomnia in 2002, with biologically based therapies (e.g., herbs) and mind-body therapies (e.g., meditation), report- 
ed as the most commonly used CAM therapies for insomnia. ${ }^{8}$ Other studies have also demonstrated that CAM is used commonly by adults with chronic medical conditions linked with insomnia. ${ }^{10,11}$ There are also emerging data to suggest that some CAM therapies, including acupressure, ${ }^{12}$ yoga, ${ }^{13}$ and Tai $\mathrm{Chi}^{14-16}$ may treat insomnia. However, the extent to which adults with insomnia symptoms use CAM is not well established.

In this context, we sought to determine the prevalence of relaxation techniques and overall CAM use, identify demographic and health-related correlates of relaxation techniques and CAM use, and to quantify reasons for use as well as the extent to which patients disclose their use to conventional medical professionals.

\section{METHODS}

\section{Data Source}

The National Health Interview Survey (NHIS) is an in-person household survey of the civilian, noninstitutionalized US population, conducted by the Census Bureau for the National Center for Health Statistics. Households are selected for faceto-face interviews in English and/or Spanish using a multistage area probability sampling design described elsewhere. ${ }^{17}$ Information on sociodemographic characteristics, health status, insurance status, and access to and use of healthcare services is collected on all household members. One adult, $\geq 18$ years, is then randomly selected from each family to provide additional detailed information on height, weight, common medical conditions, and healthcare utilization. In 2007, these sampled adults were also administered the Alternative Medicine Supplement, which queried respondents about use of relaxation techniques and more than $20 \mathrm{CAM}$ therapies. Respondents were asked, "During the past 12 months, have you used (specific therapy)?" In 2007, 23,393 sampled adults completed the Adult Questionnaire, representing a $67.8 \%$ response rate. ${ }^{18}$

\section{Insomnia Symptoms}

Insomnia symptom status was assessed based upon participants' response (yes/no) to the question, "During the past 12 months, have you regularly had insomnia or trouble sleeping?" We excluded 35 adults who responded "don't know" or refused to answer. Our final sample size included 23,358 adult respondents.

\section{Outcomes of Interest}

\section{Relaxation Techniques}

We defined relaxation techniques by use of at least one of the following practices within the past 12 months: deep breathing exercises, progressive muscle relaxation, biofeedback, and guided imagery. We selected these techniques to be consistent with the recommendations from the AASM. ${ }^{7}$

\section{Complementary and Alternative Medicine}

We also examined recent use of 4 broad CAM categories, including alternative mind-body medicine (meditation, hypnosis, qigong, Tai Chi, yoga, stress management groups); manipulative practices (chiropractic/osteopathic medicine, massage); other CAM practices (acupuncture, Ayurveda, chelation, ho- meopathy, energy healing/reiki, movement therapies, naturopathy, traditional healers), and natural products (nonvitamin, nonmineral supplements). ${ }^{9}$ Our definition did not include use of prayer, special diets (e.g., Atkins), or support groups, since these therapies are commonly excluded from epidemiologic studies of CAM use. ${ }^{10,11}$ Respondents were asked about their use of each CAM therapy during the past 12 months, except for use of natural products, which was assessed only within the previous 3 months.

We further explored use of individual relaxation techniques (deep breathing exercises, progressive muscle relaxation, guided imagery) and alternative therapies (meditation, yoga, Tai Chi, chiropractic, massage, homeopathy, acupuncture) that had a prevalence of use $\geq 1 \%$ in this sample. Among natural products, we explored use of supplements commonly promoted for treatment of insomnia, including melatonin and valerian. ${ }^{19}$

\section{Reasons for Use and Disclosure to Conventional Medical Professionals}

To assess reasons for using relaxation techniques, sample adults were first asked to report the relaxation technique that they practiced most commonly. They were then asked to respond yes/no to the following 7 questions assessing reasons for use in the past 12 months: (1) to improve or enhance energy; (2) for general wellness/general disease prevention; (3) to improve/ enhance immune function; (4) because conventional medical treatments did not help; (5) because conventional medical treatments were too expensive; (6) it was recommended by a conventional medical professional; and (7) it was recommended by family, friends, or coworkers. These 7 reasons for use were also asked for each of the other individual CAM therapies.

Next, we further examined whether adults reported using relaxation techniques and CAM specifically to treat their insomnia. For each therapy used within the past year, respondents were asked, "Did you use (specific therapy) for a specific health problem or condition?" Those responding "yes" were then queried, "For what health problem did you use (specific therapy)?" Thus, all respondents who reported having insomnia symptoms within the past year and used a specific relaxation or CAM therapy for a health problem or condition were asked whether they had used that therapy to treat their insomnia.

Finally, respondents were also asked whether they disclosed their use to medical providers. Respondents were asked, "During the past 12 months, did you let any of these conventional medical professionals know about your use of [each therapy]?" Conventional medical professionals included medical doctor (including specialists), doctor of osteopathy, nurse practitioner/ physician assistant, psychiatrist, dentist, psychologist/social worker, and pharmacist.

\section{Covariates of Interest}

We considered several factors previously found to be associated with use of relaxation techniques and CAM, and those we believed a priori to be potential correlates. ${ }^{20,21}$ As described below, these factors play different roles (predictors, confounders) in different analyses. These factors included sociodemographic characteristics, healthcare access, illness burden, and health habits. Sociodemographic characteristics included age, sex, race/ethnicity, marital status, educational attainment, imputed 
family income provided by NHIS, and region of residence. We defined health care access using several proxies, including type of insurance (uninsured, Medicare, Medicaid, private), delayed care because of worries about cost (yes/no), and delayed care because couldn't afford it (yes/no). We used indicators to capture respondents' illness burden, including perceived health status (excellent, very good, good, fair, poor), body mass index, number of emergency room visits in the past year (none, $1,>$ 1), having mobility impairment (no impairment, $\geq 1$ limitation), and self-reported history of chronic medical conditions. We considered 34 medical conditions available in NHIS, including a history of conditions related to cardiovascular, respiratory, gastrointestinal, endocrine, neurologic, rheumatologic, gynecologic, and genitourinary systems; cancer; pain syndrome; and neuropsychiatric symptoms. Indicators of health habits were smoking status (current, former, never), alcohol intake (abstain, infrequent/light, moderate, heavy consumption), ${ }^{22}$ and physical activity level (low, moderate, high). ${ }^{23}$

\section{Statistical Analyses}

We used bivariable analyses to compare adults with insomnia symptoms to those without these symptoms. We estimated the age-sex adjusted prevalence of use of relaxation techniques and CAM therapies. We further compared reasons for use and disclosure to conventional medical professionals among adults with and without insomnia symptoms.

\section{Multivariable Methods to Identify Medical Conditions as Potential Confounders}

Since few previous studies have examined the relationship between relaxation techniques use and chronic medical conditions, we first developed a multivariable logistic regression model to identify medical conditions associated with use of relaxation techniques in the past 12 months in our sample. Conditions with a p-value $<0.25$ on bivariable analyses were considered for inclusion in the multivariable model. We used a stepwise backward elimination process that adjusted for sociodemographic characteristics, insurance status, and health habits. Conditions with a Wald statistic $p$-value $\leq 0.05$ were retained in our final model. We repeated this procedure using CAM as an outcome to assess for medical conditions associated with CAM use.

\section{Multivariable Methods to Assess the Associations between Insomnia Symptoms and Relaxation Technique Use and CAM Use}

Next, we used multivariable logistic regression to assess whether insomnia symptoms are independently associated with the use of relaxation techniques, after adjusting for sociodemographic characteristics, health care access, illness burden (see Appendix for list of medical conditions), and health habits. Factors considered a priori to be important (i.e., imputed family income, insurance status) and factors with a Wald statistic $\mathrm{p}$-value $\leq 0.05$ were retained in our final model. We assessed confounding by adding each nonsignificant factor back into the model and assessed changes in the estimated $\beta$-coefficient for insomnia symptoms (our main factor of interest). Factors that changed this $\beta$-coefficient by $\geq 10 \%$ were considered confounders and retained in our final model. We also employed this strategy to build a separate model examining the associa- tions between insomnia symptoms status and CAM use (see Appendix for list of medical conditions).

\section{Multivariable Methods to Identify Predictors of Relaxation Technique and CAM Use}

We employed stepwise backward-elimination modeling procedures to identify independent correlates of relaxation techniques use in adults with insomnia symptoms adjusting for sociodemographic characteristics, health care access, medical conditions associated with relaxation techniques use, and health habits. Factors with a Wald statistic p-value $\leq 0.05$ were retained in our final model. Lastly, we repeated this modeling procedure to identify correlates of CAM use among adults with insomnia symptoms. To facilitate comparisons of predictors between the 2 outcomes, our final models included a common set of predictors that included factors which were significantly associated with either outcome. We present this common set of predictors in Tables $\mathbf{3}$ and $\mathbf{4}$ and note changes.

All analyses were performed using SAS-callable SUDAAN version 10.0 (Research Triangle Park, NC) to account for the complex sampling design, and results were weighted to reflect US national estimates. The Beth Israel Deaconess Medical Center Institutional Review Board determined this study to be exempt.

\section{RESULTS}

\section{Sample Characteristics}

Overall, 4,415 (18.1\%) of adults self-reported regularly experiencing insomnia/difficulty sleeping within the past 12 months. Table 1 presents the characteristics of adults by insomnia symptom status. Compared with adults without insomnia symptoms, those with insomnia symptoms were more often older, female, had lower educational and income levels, and had poorer health habits (i.e., smoke, have heavy alcohol consumption, and low physical activity levels).

\section{Use of Relaxation Techniques}

Table 2 lists the age-sex adjusted prevalence of relaxation techniques and CAM use by insomnia symptom status. More than 1 in 5 respondents with insomnia (22.9\%) used some form of relaxation technique annually, compared with $11.2 \%$ of adults without insomnia symptoms $(\mathrm{p}<0.0001)$. After adjustment, adults with insomnia symptoms were more likely to use relaxation techniques (adjusted odds ratio [OR] 1.42, 95\% confidence interval $[\mathrm{CI}] 1.25,1.61)$. Among relaxation techniques, deep breathing exercise was the most commonly used practice by adults with insomnia symptoms, with lower prevalence of use of progressive muscle relaxation and guided imagery.

\section{Reasons for Use and Disclosure to Conventional Medical Professionals}

Figure 1 depicts reasons for relaxation techniques use by insomnia symptom status. Respondents both with and without insomnia most frequently cited using relaxation techniques for general wellness/disease prevention. Compared with adults without insomnia, adults with insomnia symptoms were more likely using relaxation techniques to treat a specific medical 
Table 1-Characteristics of adults by insomnia symptom status $(n=23,358)$

\begin{tabular}{|c|c|c|c|c|}
\hline & $\begin{array}{l}\text { Without Insomnia ( } \mathrm{n}=18,943) \\
\mathrm{n}(\%)^{*}\end{array}$ & $\begin{array}{l}\text { With Insomnia }(n=4,415) \\
n(\%)^{*}\end{array}$ & $\begin{array}{c}\text { Prevalence of } \\
\text { Insomnia Symptoms } \\
\%^{*}\end{array}$ & $\begin{array}{c}\text { Chi-square } \\
\text { p-value }\end{array}$ \\
\hline Estimated Population Size ${ }^{\dagger}$ & $N=182,379,237$ & $N=40,426,178$ & & \\
\hline $\begin{array}{c}\text { Age (years) } \\
18-29 \\
30-39 \\
40-49 \\
50-59 \\
60-69 \\
70-79 \\
>80\end{array}$ & $\begin{array}{c}3,982(23.6) \\
3,645(18.5) \\
3,537(19.7) \\
3,004(16.3) \\
2,328(11.5) \\
1,496(6.5) \\
951(3.9)\end{array}$ & $\begin{array}{l}558(15.3) \\
668(15.0) \\
883(20.5) \\
896(20.6) \\
652(13.8) \\
449(8.9) \\
309(5.9)\end{array}$ & $\begin{array}{l}12.5 \\
15.2 \\
18.7 \\
21.9 \\
21.0 \\
23.3 \\
25.5\end{array}$ & $<0.0001$ \\
\hline $\begin{array}{l}\text { Sex } \\
\text { Male } \\
\text { Female }\end{array}$ & $\begin{array}{r}8,736(50.0) \\
10,207(50.0)\end{array}$ & $\begin{array}{l}1,616(40.2) \\
2,799(59.8)\end{array}$ & $\begin{array}{l}15.1 \\
21.0\end{array}$ & $<0.0001$ \\
\hline $\begin{array}{l}\text { Education } \\
\quad<\text { High School Graduate } \\
\text { High School Graduate } \\
\text { > High School Graduate }\end{array}$ & $\begin{array}{r}3,320(15.2) \\
5,270(28.7) \\
10,134(56.1)\end{array}$ & $\begin{array}{r}901(17.6) \\
1,245(29.9) \\
2,237(52.5)\end{array}$ & $\begin{array}{l}20.5 \\
18.8 \\
17.2\end{array}$ & $<0.001$ \\
\hline $\begin{array}{l}\text { Race/Ethnicity } \\
\text { Non-Hispanic White } \\
\text { Non-Hispanic Black } \\
\text { Hispanic } \\
\text { Asian } \\
\text { Other }\end{array}$ & $\begin{array}{c}11,050(67.7) \\
2,976(11.8) \\
3,523(13.9) \\
1,067(5.0) \\
327(1.7)\end{array}$ & $\begin{array}{r}2,840(73.8) \\
641(10.1) \\
671(11.1) \\
140(2.6) \\
123(2.4)\end{array}$ & $\begin{array}{l}19.5 \\
16.0 \\
15.0 \\
10.3 \\
23.6\end{array}$ & $<0.0001$ \\
\hline $\begin{array}{l}\text { Imputed Income } \\
\quad<\$ 20,000 \\
\$ 20,000-\$ 34,999 \\
\$ 35,000-\$ 65,000 \\
>\$ 65,000\end{array}$ & $\begin{array}{l}4,285(15.8) \\
3,580(16.5) \\
5,119(28.0) \\
5,959(39.6)\end{array}$ & $\begin{array}{r}1,436(24.0) \\
923(20.0) \\
1,038(25.5) \\
1,018(30.5)\end{array}$ & $\begin{array}{l}25.2 \\
21.2 \\
16.8 \\
14.6\end{array}$ & $<0.0001$ \\
\hline $\begin{array}{l}\text { Insurance } \\
\text { Uninsured } \\
\text { Medicare } \\
\text { Medicaid } \\
\text { Private } \\
\text { Other }\end{array}$ & $\begin{array}{l}3,313(16.8) \\
3,688(16.1) \\
1,045(4.5) \\
9,504(55.2) \\
1,333(7.4)\end{array}$ & $\begin{aligned} 728(16.4) \\
1,296(26.3) \\
418(8.0) \\
1,663(42.0) \\
298(7.3)\end{aligned}$ & $\begin{array}{l}17.7 \\
26.6 \\
28.0 \\
14.4 \\
18.1\end{array}$ & $<0.0001$ \\
\hline $\begin{array}{l}\text { Region } \\
\text { Northeast } \\
\text { Midwest } \\
\text { South } \\
\text { West }\end{array}$ & $\begin{array}{l}3,217(17.3) \\
4,253(24.4) \\
6,992(36.3) \\
4,481(22.0)\end{array}$ & $\begin{array}{r}702(16.2) \\
953(22.7) \\
1,710(38.3) \\
1,050(22.9)\end{array}$ & $\begin{array}{l}17.1 \\
17.0 \\
19.0 \\
18.7\end{array}$ & $<0.001$ \\
\hline $\begin{array}{l}\text { Marital Status } \\
\text { Married } \\
\text { Widowed } \\
\text { Divorced or separated } \\
\text { Never Married }\end{array}$ & $\begin{array}{c}10,032(63.7) \\
1,676(5.7) \\
2,780(9.6) \\
4,341(21.0)\end{array}$ & $\begin{array}{c}1,955(56.7) \\
582(9.0) \\
999(16.3) \\
868(18.0)\end{array}$ & $\begin{array}{l}16.5 \\
26.0 \\
27.4 \\
16.0\end{array}$ & $<0.0001$ \\
\hline $\begin{array}{l}\text { Smoking Status } \\
\text { Current } \\
\text { Former } \\
\text { Never }\end{array}$ & $\begin{array}{r}3,238(18.1) \\
3,771(20.4) \\
11,601(61.5)\end{array}$ & $\begin{array}{l}1,130(27.4) \\
1,151(26.4) \\
2,075(46.2)\end{array}$ & $\begin{array}{l}25.2 \\
22.3 \\
14.3\end{array}$ & $<0.0001$ \\
\hline
\end{tabular}

$\mathrm{n}=$ sample size; $\mathrm{N}=$ Population estimate. ${ }^{\dagger}$ NHIS complex sampling scheme allows for estimates of U.S. population. *\%, estimate weighted to reflect population. ${ }^{\ddagger}$ Alcohol intake: abstainer $(<12$ drinks in lifetime), rare $(<1$ drink/month in past year), light ( $\leq 3$ drinks/week), moderate $(>3$ and $\leq 7$ drinks/ week for women, $>3$ and $\leq 14$ drinks/week for men), or heavy (> 7 drinks/week for women and $>14$ drinks/week for men). \$Physical activity levels: Vigorous = vigorous activity 2 times/week or moderate activity 4 times/week; moderate = vigorous activity 1 time/week or moderate activity $1-3$ times/ week, sedentary $=$ no vigorous or moderate activity/week. 
Table 1 (continued)-Characteristics of adults by insomnia symptom status $(n=23,358)$

\begin{tabular}{|c|c|c|c|c|}
\hline & $\begin{array}{l}\text { Without Insomnia }(n=18,943) \\
n(\%)^{*}\end{array}$ & $\begin{array}{c}\text { With Insomnia }(n=4,415) \\
n(\%)^{*}\end{array}$ & $\begin{array}{c}\text { Prevalence of } \\
\text { Insomnia Symptoms } \\
\%^{*}\end{array}$ & $\begin{array}{c}\text { Chi-square } \\
\text { p-value }\end{array}$ \\
\hline Alcohol Intake $\ddagger$ & & & & $<0.0001$ \\
\hline Abstainer & $7,463(38.7)$ & $1,759(38.7)$ & 18.3 & \\
\hline Light & $7,366(41.6)$ & $1,776(42.7)$ & 18.7 & \\
\hline Moderate & $2,491(14.8)$ & $498(12.3)$ & 15.7 & \\
\hline Heavy & $843(4.9)$ & $263(6.2)$ & 22.3 & \\
\hline Physical Activity Level\$ $\$$ & & & & $<0.0001$ \\
\hline Low & $7,940(39.9)$ & $2,104(47.0)$ & 21.0 & \\
\hline Moderate & $2,879(16.4)$ & $692(15.7)$ & 17.6 & \\
\hline High & $7,755(43.7)$ & $1,562(37.3)$ & 16.0 & \\
\hline \multicolumn{5}{|l|}{ Neuropsychiatric Symptoms } \\
\hline Mania/psychosis & $65(0.3)$ & $91(2.3)$ & 64.0 & $<0.0001$ \\
\hline Frequently anxious & $1,083(5.7)$ & $1,514(34.1)$ & 57.7 & $<0.0001$ \\
\hline Excessive daytime sleepiness & $933(4.9)$ & $1,482(34.2)$ & 60.6 & $<0.0001$ \\
\hline Attention deficit disorder & $339(2.3)$ & $217(5.7)$ & 35.9 & $<0.0001$ \\
\hline \multicolumn{5}{|l|}{ Pain Syndromes } \\
\hline Dental pain/gum disease & $2,077(11.3)$ & $1,172(25.4)$ & 33.3 & $<0.0001$ \\
\hline Neck pain & $1,784(9.4)$ & $1,319(29.5)$ & 41.1 & $<0.0001$ \\
\hline Jaw/ear pain & $493(2.7)$ & $465(10.2)$ & 45.5 & $<0.0001$ \\
\hline Low back pain & $3,858(20.3)$ & $2,205(49.3)$ & 35.0 & $<0.0001$ \\
\hline \multicolumn{5}{|l|}{ Other Medical Conditions } \\
\hline Hypertension & $4,894(24.0)$ & $1,942(41.2)$ & 27.6 & $<0.0001$ \\
\hline Asthma & $1,742(9.3)$ & $812(18.5)$ & 30.1 & $<0.0001$ \\
\hline Heartburn & $3,244(17.5)$ & $1,798(41.1)$ & 34.3 & $<0.0001$ \\
\hline Musculoskeletal strain & $904(5.1)$ & $605(14.7)$ & 39.1 & $<0.0001$ \\
\hline
\end{tabular}

$\mathrm{n}=$ sample size; $\mathrm{N}=$ Population estimate. ${ }^{\dagger} \mathrm{NHIS}$ complex sampling scheme allows for estimates of U.S. population. ${ }^{*} \%$, estimate weighted to reflect population. ${ }^{\ddagger}$ Alcohol intake: abstainer ( $<12$ drinks in lifetime), rare ( $<1$ drink/month in past year), light ( $\leq 3$ drinks/week), moderate $(>3$ and $\leq 7$ drinks/

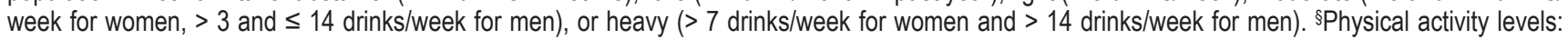
Vigorous = vigorous activity 2 times/week or moderate activity 4 times/week; moderate = vigorous activity 1 time/week or moderate activity 1-3 times/ week, sedentary $=$ no vigorous or moderate activity/week.

condition, because a conventional medical professional recommended it, because conventional medical treatments did not help, and because medical treatments were too expensive. Fewer than $20 \%$ of all adults (19.1\%) disclosed use of relaxation techniques to conventional medical professionals; adults with insomnia symptoms disclosed use of relaxation techniques to their providers more often than those without insomnia $(26.3 \%$ vs. $15.7 \% ; \mathrm{p}<0.0001)$. Although $29.9 \%(\mathrm{n}=291)$ of adults with insomnia reported using relaxation techniques for a specific medical condition, fewer than 30 adults in our sample subsequently responded using a relaxation technique specifically for insomnia. This small sample size precluded analysis of population estimates for use of relaxation techniques for specific treatment of insomnia.

\section{Correlates of Use of Relaxation Techniques among Adults with Insomnia Symptoms}

Among adults with insomnia symptoms, factors significantly associated with relaxation techniques use included moderate alcohol consumption, having mania/psychosis, excessive daytime sleepiness, anxiety, dental pain, neck pain, jaw pain, and asthma (Table 3). Factors significantly associated with a lower likelihood of relaxation technique use included male sex, lower educational attainment, family income $<\$ 20,000$, low physical activity level, living in the South, and having hypertension. Of note, although jaw pain was a significant independent predictor of relaxation techniques, when factors independently associated with overall CAM use were added to the final model for comparison, jaw pain was no longer significant as shown in Table 3. The most likely explanation for the shift to non-significance observed in these factors is high correlations between variables.

\section{Use of Complementary and Alternative Medicine (CAM)}

Forty-five percent $(45.2 \%)$ of adults with insomnia symptoms reported using $\geq 1$ CAM therapy in the past year, compared with $30.9 \%$ of adults without insomnia ( $<<0.0001$; Table 2$)$. After adjustment, adults with insomnia symptoms were more likely to use overall CAM (aOR 1.29, 95\% CI [1.15, 1.44]). Adults with insomnia symptoms also used most categories of CAM (i.e., alternative mind-body medicine, natural products, manipulative practices, and other CAM practices) more than those without insomnia. Among individual CAM therapies, natural products were the most commonly used by adults with insomnia, followed by manipulative practices. The sleep-related natural products, melatonin and valerian, were used more frequently by adults 
Table 2-Age-sex adjusted prevalence of relaxation technique and CAM Use by insomnia status, within past 12 months

\begin{tabular}{|c|c|c|c|}
\hline Therapy & $\begin{array}{l}\text { With Insomnia Symptoms, } \\
\qquad(\mathrm{n}=4,415)\end{array}$ & $\begin{array}{l}\text { Without Insomnia Symptoms, } \\
\qquad(\mathrm{n}=18,943)\end{array}$ & $\begin{array}{l}\text { Chi-square } \\
\text { p-value }\end{array}$ \\
\hline Relaxation Techniques & $997(22.9)$ & $2,082(11.2)$ & $<0.0001$ \\
\hline Deep breathing exercises & $939(21.5)$ & $1,977(10.6)$ & $<0.0001$ \\
\hline Progressive muscle relaxation & $210(5.2)$ & $434(2.4)$ & $<0.0001$ \\
\hline Guided imagery & $152(3.6)$ & $329(1.8)$ & $<0.0001$ \\
\hline Overall CAM & $1,936(45.2)$ & $5,615(30.9)$ & $<0.001$ \\
\hline Alternative Mind-Body Medicine & $879(20.7)$ & $2,256(12.0)$ & $<0.0001$ \\
\hline Meditation & $678(16.0)$ & $1,476(7.8)$ & $<0.0001$ \\
\hline Yoga & $289(6.9)$ & $1,053(5.8)$ & 0.07 \\
\hline Tai chi & $60(1.4)$ & $207(1.0)$ & 0.056 \\
\hline Natural Products* & $1,059(24.4)$ & $2,920(16.2)$ & $<0.0001$ \\
\hline Melatonin & $64(1.5)$ & $75(0.4)$ & $<0.0001$ \\
\hline Valerian & $43(1.0)$ & $46(0.3)$ & $<0.0001$ \\
\hline Manipulative Practices & 766 (18.2) & $2,374(13.2)$ & $<0.0001$ \\
\hline Chiropractic & $464(11.0)$ & $1,389(7.6)$ & $<0.0001$ \\
\hline Massage & $467(11.0)$ & $1,363(7.5)$ & $<0.0001$ \\
\hline Other CAM Practices & $270(6.0)$ & $620(3.2)$ & $<0.0001$ \\
\hline Homeopathy & 121 (2.7) & 174 (1.2) & $<0.0001$ \\
\hline Acupuncture & $104(2.5)$ & $240(1.2)$ & $<0.0001$ \\
\hline
\end{tabular}

Values are $\mathrm{n}$ (weighted populations\%); CAM, Complementary and Alternative Medicine. *Natural products assessed within past three months. Not presented because overall prevalence $<1 \%$ or $n<50$ : Alexander Technique, Ayurveda, Biofeedback, Chelation, Energy Healing Therapy, Feldenkrais, Hypnosis, Naturopathy, Qigong, Stress Management Group, Curandero, Espiritista, Hierbero or Yerbera, Shaman, Native American Healer, Sobador.

Figure 1-Reasons for relaxation technique use by insomnia symptom status

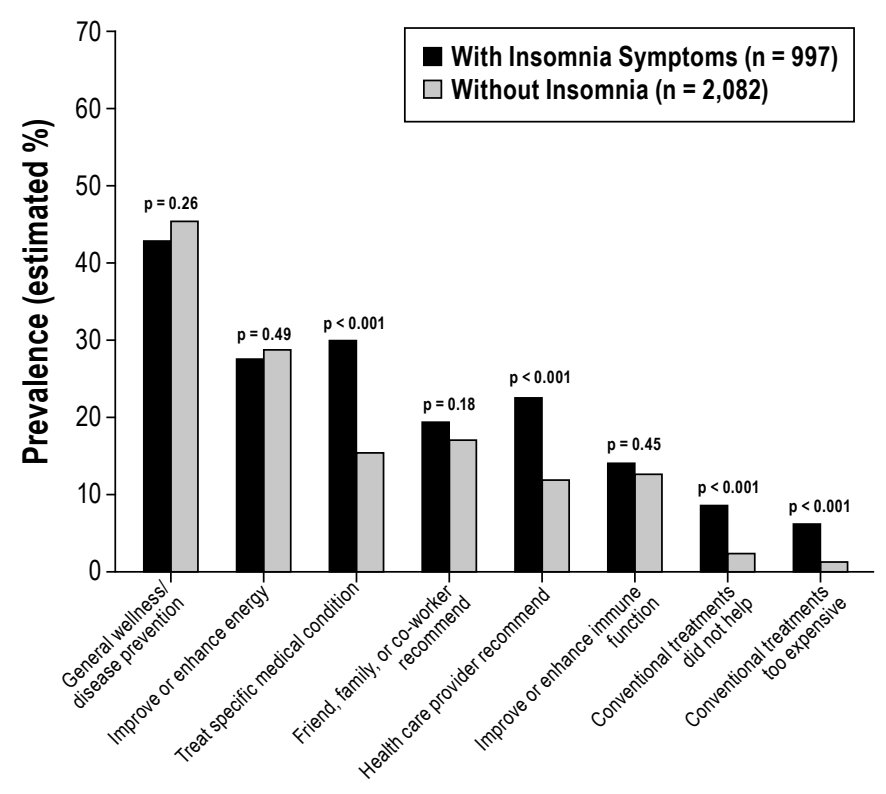

with insomnia; however, overall use of these natural products was very low. Small sample sizes $(n<30)$ precluded analysis of individual natural products for treatment of insomnia.

\section{Reasons for CAM Use and Disclosure to Conventional Medical Professionals}

Similar to use of relaxation techniques, both adults with and without insomnia symptoms most commonly cited high preva-
Figure 2-Reasons for CAM use by insomnia symptom status

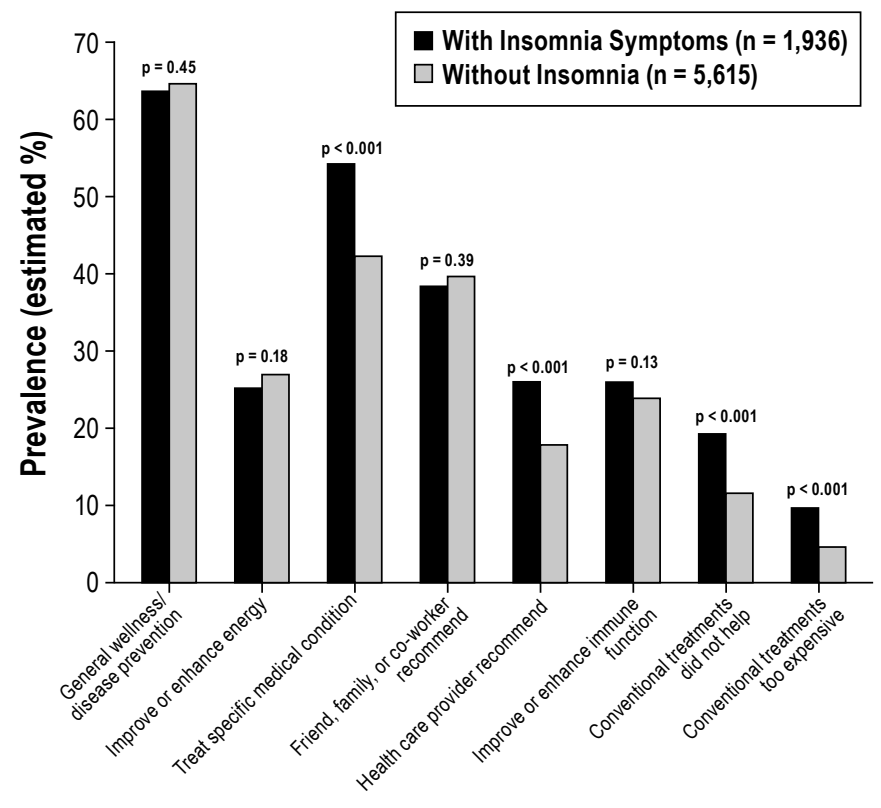

lence of CAM use for general wellness/disease prevention (Figure 2). Adults with insomnia symptoms more often cited CAM use to treat a specific medical condition, because a health care professional recommended it, because conventional medical treatments did not help, and because medical treatments were too expensive, compared with adults without insomnia. Adults with insomnia also disclosed their CAM use to conventional medical professionals more frequently than adults with- 
Table 3-Independent correlates of relaxation techniques use among adults with insomnia symptoms ( $n=4,232)^{*}$

\begin{tabular}{|c|c|c|}
\hline \multicolumn{3}{|l|}{$\begin{array}{l}\text { Factors } \\
\text { Sociodemographics }\end{array}$} \\
\hline \multicolumn{3}{|l|}{ Sex } \\
\hline Male & 0.54 & $0.44,0.67$ \\
\hline Female & 1.00 (reference) & \\
\hline \multicolumn{3}{|l|}{ Race } \\
\hline Non-Hispanic White & 1.00 (reference) & \\
\hline Non-Hispanic Black & 0.65 & $0.51,0.82$ \\
\hline Hispanic & 0.64 & $0.48,0.84$ \\
\hline Asian & 1.04 & $0.64,1.69$ \\
\hline Other & 1.42 & $0.75,2.66$ \\
\hline \multicolumn{3}{|l|}{ Education } \\
\hline$<$ High School Graduate & 0.44 & $0.31,0.63$ \\
\hline High School Graduate & 0.67 & $0.53,0.85$ \\
\hline > High School Graduate & 1.00 (reference) & \\
\hline \multicolumn{3}{|l|}{ Income } \\
\hline$<\$ 20,000$ & 0.66 & $0.49,0.89$ \\
\hline$\$ 20,000-\$ 34,999$ & 0.77 & $0.57,1.05$ \\
\hline$\$ 35,000-\$ 65,000$ & 0.90 & $0.67,1.20$ \\
\hline$>\$ 65,000$ & 1.00 (reference) & \\
\hline \multicolumn{3}{|l|}{ Region } \\
\hline Northeast & 1.24 & $0.93,1.65$ \\
\hline Midwest & 1.61 & $1.27,2.04$ \\
\hline South & 1.00 (reference) & \\
\hline West & 1.48 & $1.15,1.90$ \\
\hline \multicolumn{3}{|l|}{ Health Habits } \\
\hline \multicolumn{3}{|l|}{ Physical Activity Level } \\
\hline Low & 0.51 & $0.41,0.64$ \\
\hline Moderate & 1.08 & $0.84,1.39$ \\
\hline High & 1.00 (reference) & \\
\hline \multicolumn{3}{|l|}{ Alcohol } \\
\hline Abstainer & 1.00 (reference) & \\
\hline Light & 1.21 & $0.98,1.52$ \\
\hline Moderate & 1.46 & $1.08,1.98$ \\
\hline Heavy & 0.85 & $0.56,1.28$ \\
\hline \multicolumn{3}{|l|}{ Smoking Status } \\
\hline Current & 0.77 & $0.61,0.99$ \\
\hline Former & 1.24 & $1.03,1.50$ \\
\hline Never & 1.00 (reference) & \\
\hline \multicolumn{3}{|l|}{ Medical Conditions } \\
\hline Hypertension, history & 0.78 & $0.63,0.96$ \\
\hline Asthma, history & 1.45 & $1.17,1.81$ \\
\hline Heartburn, past 12 months & 1.02 & $0.83,1.24$ \\
\hline Dental pain, past 12 months & 1.22 & $1.01,1.49$ \\
\hline Neck pain, past 3 months & 1.38 & $1.11,1.71$ \\
\hline Jaw pain, past 3 months & 1.32 & $0.98,1.78$ \\
\hline Low back pain, past 3 months & 1.11 & $0.91,1.35$ \\
\hline Mania/psychosis, history & 2.68 & $1.39,5.14$ \\
\hline Anxiety, past 12 months & 1.71 & $1.42,2.19$ \\
\hline Excessive daytime sleepiness, past 12 months & 1.28 & $1.06,1.55$ \\
\hline Attention Deficit Disorder, history & 1.27 & $0.79,2.06$ \\
\hline Musculoskeletal strain, past 12 months & 1.27 & $0.94,1.73$ \\
\hline
\end{tabular}

Bold denotes significant predictors. ${ }^{*}$ Observations with complete data. ${ }^{\dagger}$ Adjusted for sociodemographic factors, health care access, and health habits. 
out insomnia (47.4\% vs. $40.7 \%$, p $<0.0001$ ). While $54.2 \%$ of adults $(n=1,040)$ with insomnia reported using a CAM therapy for a specific health problem or condition, the reported conditions were usually not insomnia. Only $1.8 \%(n=82)$ reported using a CAM therapy for specific treatment of their insomnia.

\section{Correlates of CAM Use among Adults with Insomnia Symptoms}

Among adults with insomnia symptoms, factors significantly associated with CAM use included a former smoking history, having attention deficit disorder, heartburn, severe musculoskeletal sprains or strains, dental pain, neck pain, or low back pain during the past 3 months (Table 4). Factors significantly associated with a lower likelihood of relaxation techniques use included male sex, Hispanic or Non-Hispanic black race, lower educational attainment, family income $<\$ 35,000$, low physical activity level, living in the South, currently smoking, and having hypertension. While musculoskeletal strain was a significant independent predictor of CAM use, it no longer achieved statistical significance once factors associated with relaxation use were included in the model.

\section{DISCUSSION}

We found that greater than 1 in 5 adults with insomnia symptoms used relaxation techniques, and $45 \%$ of adults with insomnia symptoms used at least one CAM therapy in the past year. Although adults with insomnia symptoms had higher likelihood of use of both relaxation techniques and CAM compared with adults without insomnia, fewer than $2 \%$ of adults with insomnia symptoms said that they used CAM to treat their insomnia. Hence, although adults with insomnia are more likely to use relaxation techniques and CAM and have higher use for specific medical conditions, their use is not driven by treatment of insomnia symptoms. General wellness/disease prevention was the most frequently cited reason for use of both relaxation techniques and CAM. Only $26 \%$ of adults with insomnia symptoms reported that they had told their conventional providers about their use of relaxation techniques, while $47 \%$ had disclosed their use of CAM. Among adults with insomnia symptoms, factors associated with lower likelihood of relaxation techniques use were being male, having lower educational attainment, low physical activity, family income $<\$ 20,000$, living in the South, and having a history of hypertension.

To our knowledge, this is the first study to exclusively examine the national prevalence of relaxation techniques by adults with insomnia symptoms in the United States. Relaxation training has been included in the AASM practice parameters as a standard therapy in the treatment of chronic insomnia since 2006. ${ }^{7}$ While we found that relaxation techniques are commonly used by adults with insomnia symptoms, we also found that very few patients are using relaxation techniques to specifically treat their insomnia. It is unclear why use of relaxation training for insomnia is uncommon, but may relate to factors at the practitioner and systems levels. ${ }^{24}$ For example, there is a limited supply of behavioral sleep medicine practitioners and relatively few sleep medicine clinics have fully integrated behavioral sleep medicine services. ${ }^{25}$ There may also be lingering questions regarding the efficacy of relaxation techniques given their generally weaker effect sizes compared with stimulus control and multi-component CBT-I therapies for insomnia, ${ }^{23,24}$ and further challenges related to current reimbursement models for behavioral therapies. ${ }^{25}$

Additionally we found that nearly $75 \%$ of adults with insomnia symptoms using relaxation techniques did not disclose their use to a conventional medical professional. Patients' nondisclosure of relaxation techniques use may represent a missed opportunity for providers to discuss patients' interest in and experience with relaxation techniques, particularly in relation to insomnia. However, it is unclear why patients do not tell their providers about their use of relaxation techniques. Given the potential under use of relaxation techniques for this prevalent condition, more research is needed to understand the barriers to implementation of targeted relaxation therapies for patients with insomnia.

Alternative mind-body therapies such as meditation and Tai Chi, represent a diverse group of meditative practices, thought to evoke similar physiologic pathways to traditional relaxation training, ${ }^{26}$ and have been purported as treatments for insomnia. ${ }^{27}$ Nascent research in this area has been promising, though results vary by individual therapy. For example, despite popularized claims of the therapeutic benefit for meditation for sleep, data from randomized trials evaluating mindfulness meditation for sleep disturbances are mixed. ${ }^{28-33}$ Conversely, three recent randomized controlled studies evaluating the benefit of Tai Chi for sleep have demonstrated improvement in subjective sleep parameters (i.e., Pittsburgh Sleep Quality Index [PSQI]). ${ }^{14-16} \mathrm{How}-$ ever, larger randomized controlled trials with objective outcome measures are needed to improve our understanding of the efficacy of alternative mind-body therapies, with particular attention given to important methodological issues that are related to behavioral interventions, such as difficulty with blinding.

While we found that nearly one in four adults with insomnia symptoms had used natural products within the past three months, we were surprised to find low prevalence of use of valerian and melatonin, which are natural products promoted specifically for their hypnotic effects. It is possible that the observed low use of these sleep-related supplements may reflect the recent findings of two large randomized controlled trials, that failed to demonstrate improvement in subjective sleep parameters among patients with insomnia taking valerian compared with placebo. ${ }^{34,35}$ Similarly, recent reviews on melatonin for treatment of primary insomnia failed to show robust improvements, particularly when accounting for circadian rhythm disorders. ${ }^{36}$ Despite low prevalence of use, it remains important to query patients about their use of natural products, since potential interactions exist between some natural products (e.g., valerian, melatonin) and commonly prescribed hypnotics. This is particularly important given that many patients with insomnia do not disclose their CAM use to conventional medical professionals.

\section{Limitations}

The NHIS is a cross-sectional survey that relies on self-report, with potential for misclassification and recall bias. This is of particular concern since insomnia symptoms can be episodic, and participants may not report having had insomnia within the past year if their symptoms have remitted at the time of their interview, but may report having used relaxation techniques 
Table 4-Independent correlates of CAM use among adults with insomnia symptoms $(n=4,230)^{*}$

Factors

Sociodemographics

Sex

Male

Female

\section{Race}

Non-Hispanic White

Non-Hispanic Black

Hispanic

Asian

Other

\section{Education}

$<$ High School Graduate

High School Graduate

$>$ High School Graduate

\section{Income \\ $<\$ 20,000$ \\ $\$ 20,000-\$ 34,999$ \\ $\$ 35,000-\$ 65,000$ \\ $>\$ 65,000$}

Region

Northeast

Midwest

South

West

Health Habits
Adjusted Odds Ratio ${ }^{\dagger}$

0.68

1.00 (reference)

1.00 (reference)

0.66

0.66

1.05

1.36

0.40

0.65

1.00 (reference)

0.67

0.68

0.83

1.00 (reference)

1.06

1.28

1.00 (reference)

1.55
95\% Confidence Interval

$0.57,0.80$

$0.52,0.84$

$0.48,0.83$

$0.65,1.69$

$0.72,2.60$

$0.31,0.52$

$0.53,0.78$

$0.53,0.86$

$0.52,0.88$

$0.66,1.05$

$0.84,1.33$

$1.00,1.62$

$1.25,1.92$

$0.50,0.71$

$0.88,1.37$

1.09

1.00 (reference)

1.00 (reference)

1.32

1.61

1.42

1.10, 1.59

$1.19,2.18$

$1.02,1.97$

0.76

1.25

1.00 (reference)

$0.60,0.97$

$1.03,1.51$

0.81

1.10

1.22

1.34

1.63

1.21

1.27

1.01

1.20

1.01

1.57

1.32
$0.69,0.95$

$0.88,1.38$

$1.02,1.45$

1.15, 1.62

$1.32,2.01$

$0.89,1.64$

1.06, 1.52

$0.49,2.06$

$0.82,1.23$

$1.08,2.30$

$0.99,1.76$

Bold denotes significant predictors. *Observations with complete data. ${ }^{\dagger}$ Adjusted for sociodemographic factors, health care access and health habits. 
or CAM to alleviate their insomnia symptoms. Similarly, misclassification may have occurred if a respondent reported using relaxation techniques or CAM therapy to treat a medical condition associated with insomnia, even if it they had use it to improve their comorbid insomnia symptoms. Another important limitation is that insomnia was not defined using established diagnostic criteria, and therefore we were not able to distinguish between insomnia symptoms and insomnia as a clinical condition. Furthermore, NHIS does not assess quantity or duration of relaxation technique or CAM use, which limits our ability to distinguish the characteristics of one-time users to those adults who practice relaxation techniques and CAM regularly; nor were we able to distinguish whether instructions for relaxation techniques use were provided by a health care professional or self-taught. Lastly, the 2007 NHIS was administered only in English and Spanish, and certain immigrant populations that are less acculturated may have different patterns of use that were not captured. ${ }^{37}$ Despite these inherent limitations, our findings are based upon the most recent nationally representative data available on relaxation techniques and CAM use among adults with insomnia symptoms in the United States.

\section{CONCLUSION}

In summary, we found that despite high prevalence of use of relaxation techniques and CAM by adults with insomnia symptoms in the United States, few adults used relaxation techniques to treat their insomnia. Only one in four adults disclosed use of relaxation techniques to their conventional medical professionals. Better understanding of the barriers of relaxation techniques use and a refinement in our understanding of their efficacy and neurobiologic underpinnings would broaden our ability to provide targeted behavioral therapies to patients with sleep disturbances.

\section{REFERENCES}

1. Ohayon MM. Epidemiology of insomnia: what we know and what we still need to learn. Sleep Med Rev 2002; 6:97-114.

2. Bonnet MH, Arand DL. Hyperarousal and insomnia: state of the science. Sleep Med Rev 2010;14:9-15.

3. Winkelman JW, Buxton OM, Jensen JE, et al. Reduced brain GABA in primary insomnia: preliminary data from $4 \mathrm{~T}$ proton magnetic resonance spectroscopy (1H-MRS). Sleep 2008;31:1499-506.

4. Kahn M, Baker BL. Weiss JM. Treatment of insomnia by relaxation training. J Abnorm Psychol 1968; 73:556-8.

5. Borkovec TD, Fowles DC. Controlled investigation of the effects of progressive and hypnotic relaxation on insomnia. J Abnorm Psychol 1973;82:153-8.

6. Nicassio P, Bootzin R. A comparison of progressive relaxation and autogenic training as treatments for insomnia. J Abnorm Psychol 1974; 83:253-60.

7. Morgenthaler T, Kramer M, Alessi C, et al. Practice parameters for the psychological and behavioral treatment of insomnia: an update. An American Academy of Sleep Medicine report. Sleep 2006;29:1415-9.

8. Pearson NJ, Johnson LL, Nahin RL. Insomnia, trouble sleeping, and complementary and alternative medicine: Analysis of the 2002 national health interview survey data. Arch Intern Med 2006;166:1775-82.

9. What Is Complementary and Alternative Medicine? National Center for Complementary and Alternative Medicine.Available from: http://nccam.nih.gov/health/ whatiscam/\#types, accessed April 20, 2011.

10. Wells RE, Phillips RS, Schachter SC, McCarthy EP. Complementary and alternative medicine use among US adults with common neurological conditions. $J$ Neurol 2010;257:1822-31

11. Bertisch SM, Wee CC, Phillips RS, McCarthy EP. Alternative mind-body therapies used by adults with medical conditions. J Psychosom Res 2009;66:511-9.
12. Sarris J, Byrne GJ. A systematic review of insomnia and complementary medicine. Sleep Med Rev 2011;15:99-106.

13. Khalsa SB. Treatment of chronic insomnia with yoga: a preliminary study with sleep-wake diaries. Appl Psychophysiol Biofeedback 2004;29:269-78.

14. Irwin MR, Olmstead R, Motivala SJ. Improving sleep quality in older adults with moderate sleep complaints: A randomized controlled trial of Tai Chi Chih. Sleep 2008;31:1001-8.

15. Wang $\mathrm{C}$, Schmid $\mathrm{CH}$, Rones $\mathrm{R}$, et al. A randomized trial of tai chi for fibromyalgia. N Engl J Med 2010;363:743-54.

16. Li F, Fisher KJ, Harmer P, Irbe D, Tearse RG, Weimer C. Tai chi and self-rated quality of sleep and daytime sleepiness in older adults: a randomized controlled trial. J Am Geriatr Soc 2004;52:892-900.

17. Centers for Disease Control. 2006, 2007 National Health Interview Survey (NHIS) Survey Description Documents, Appendices III and VII: Variance Estimation and Other Analytic Issues. Available from: http://www.cdc.gov/nchs/data/ nhis/2006_2007var.pdf, accessed April 20, 2011.

18. National Center for Health Statistics. 2007 National Health Interview Survey (NHIS) Survey Description Document. Available from: ftp://ftp.cdc.gov/pub/ Health_Statistics/NCHS/Dataset_Documentation/NHIS/2007/srvydesc.pdf, accessed April 20, 2011

19. Bliwise DL, Ansari FP. Insomnia associated with valerian and melatonin usage in the 2002 National Health Interview Survey. Sleep 2007;30:881-4.

20. Eisenberg DM, Kessler RC, Foster C, Norlock FE, Calkins DR, Delbanco TL. Unconventional medicine in the United States. Prevalence, costs, and patterns of use. N Engl J Med 1993;328:246-52.

21. Wolsko PM, Eisenberg DM, Davis RB, Ettner SL, Phillips RS. Insurance coverage, medical conditions, and visits to alternative medicine providers: results of a national survey. Arch Intern Med 2002;162:281-7.

22. Health, United States, 2007. U.S. Department Health and Human Services, Center for Disease Control and Prevention, National Center for Health Statistics. Available from: http://www.cdc.gov/nchs/data/hus/hus07.pdf, accessed April 20, 2011.

23. Kushi LH, Fee RM, Folsom AR, Mink PJ, Anderson KE, Sellers TA. Physical activity and mortality in postmenopausal women. JAMA 1997;277:1287-92.

24. Perlis ML, Smith MT. How can we make CBT-I and other BSM services widely available? J Clin Sleep Med 2008;4:11-3.

25. Pigeon WR, Crabtree VM, Scherer MR. The future of behavioral sleep medicine. J Clin Sleep Med 2007:3:73-9.

26. Ospina MB, Bond K, Karkhaneh M, et al. Meditation practices for health: state of the research. Evid Rep Technol Assess (Full Rep) 2007:1-263.

27. Britton W. Meditation for Sleep: Paradoxes and Promises. 2010 Jun In: Huffington Post. HuffPost Living [Internet]. Available from: http://www. huffingtonpost.com/willoughby-britton/sleep-tips-meditation-for_b_597600. html, accessed April 20, 2011.

28. Britton WB, Haynes PL, Fridel KW, Bootzin RR. Polysomnographic and subjective profiles of sleep continuity before and after mindfulness-based cognitive therapy in partially remitted depression. Psychosom Med 2010;72:539-48.

29. Shapiro SL, Bootzin RR, Figueredo AJ, Lopez AM, Schwartz GE. The efficacy of mindfulness-based stress reduction in the treatment of sleep disturbance in women with breast cancer: an exploratory study. J Psychosom Res 2003;54:85-91.

30. Gross CR, Kreitzer MJ, Thomas W, et al. Mindfulness-based stress reduction for solid organ transplant recipients: a randomized controlled trial. Altern Ther Health Med 2010;16:30-8.

31. Gross CR, Kreitzer MJ, Reilly-Spong M, et al. Mindfulness-based stress reduction versus pharmacotherapy for chronic primary insomnia: a randomized controlled clinical trial. Explore (NY) 2011;7:76-87.

32. Goldenberg DL, Kaplan KH, Nadeau MG, Brodeur C, Smith S, Schmid CH. A controlled trial of a stress-reduction, cognitive behavioral treatment program in fibromyalgia. J Musculoskel Pain 1994;2:53-6.

33. Roth $B$, Robbins D. Mindfulness-based stress reduction and health-related quality of life: findings from a bilingual inner-city patient population. Psychosom Med 2004;66:113-23.

34. Fernandez-San-Martin MI, Masa-Font R, Palacios-Soler L, Sancho-Gomez P, Calbo-Caldentey C, Flores-Mateo G. Effectiveness of Valerian on insomnia: a meta-analysis of randomized placebo-controlled trials. Sleep Med 2010;11:505-11.

35. Bent S, Padula A, Moore D, Patterson M, Mehling W. Valerian for sleep: a systematic review and meta-analysis. Am J Med 2006;119:1005-12.

36. Buscemi N, Vandermeer B, Hooton N, et al. The efficacy and safety of exogenous melatonin for primary sleep disorders. A meta-analysis. J Gen Intern Med 2005;20:1151-8.

37. Hsiao AF, Wong MD, Goldstein MS, et al. Variation in complementary and alternative medicine (CAM) use across racial/ethnic groups and the development of ethnic-specific measures of CAM use. J Altern Complement Med 2006;12:281-90. 


\section{ACKNOWLEDGMENTS}

The authors thank Julie K. Smith for her thoughtful editorial review. Work for this study was conducted at Beth Israel Deaconess Medical Center, Boston, MA. Dr. Bertisch was supported by a K23 Career Development Award (K23AT005104). Dr. Wells was supported by an institutional National Research Service Award (T32AT000051) from the National Center for Complementary \& Alternative Medicine (NCCAM) at the National Institutes of Health, USA. Dr. Smith was supported by the National Institute of Arthritis and Musculoskeletal Disease (R01s AR054871 and AR059410) Dr. McCarthy was supported by R03AT002236, also from NCCAM. The content is solely the responsibility of the authors and does not necessarily represent the official views of the National Center for Complementary \& Alternative Medicine or the National Institutes of Health, USA.

\section{SUBMISSION \& CORRESPONDENCE INFORMATION}

Submitted for publication June, 2011

Submitted in final revised form May, 2012

Accepted for publication May, 2012

Address correspondence to: Suzanne M. Bertisch, M.D., M.P.H., Divisions of General Medicine and Primary Care, and Pulmonary, Critical Care, and Sleep Medicine, Department of Medicine, Beth Israel Deaconess Medical Center, Harvard Medical School, 1309 Beacon Street, 2nd Floor, Brookline, MA 02446; Tel: (617)-754-1404; Fax: (617)-754-1440; E-mail: sbertisc@caregroup.harvard.edu

\section{DISCLOSURE STATEMENT}

This was not an industry supported study. Dr. Smith is an owner and consultant to B-Med Technologies, Inc. The terms of this arrangement are being managed by the Johns Hopkins University in accordance with its conflict of interest policies. The other authors have indicated no financial conflicts of interest.

\section{APPENDIX}

1. Medical conditions included in multivariable model assessing the association between insomnia symptoms and relaxation technique use: cardiovascular disease, chronic obstructive pulmonary disease, asthma, hayfever/sinusitis, excessive daytime sleepiness, anxiety, bipolar disorder, mania/psychosis, dental pain, arthritis, neck pain, low back pain, jaw/ear pain, musculoskeletal strain/sprain

2. Medical conditions included in multivariable model assessing the association between insomnia symptoms and CAM use: hayfever/sinusitis, asthma, heartburn, excessive daytime sleepiness, anxiety, bipolar disorder, dental pain, arthritis, neck pain, low back pain, jaw/ear pain 